\title{
Spectrum of Signals on the Quaternion Fourier Transform Domain
}

\author{
Guicheng Yang', Yingxiong $\mathrm{Fu}^{{ }^{2 *}}$ \\ ${ }^{1}$ Business College, Hubei University of Technology, Wuhan, China \\ ${ }^{2}$ Faculty of Mathematics and Computer Science, Hubei University, Wuhan, China \\ Email: *834082116@qq.com, fyx@hubu.edu.cn
}

Received August 2013

\begin{abstract}
The quaternion Fourier transform plays a vital role in the representation of two-dimensional signals. This paper characterizes spectrum of quaternion-valued signals on the quaternion Fourier transform domain by the partial derivative.
\end{abstract}

Keywords: Spectrum; Quaternion Fourier Transform; Partial Derivative

\section{Introduction}

The quaternion Fourier transform (QFT) is a nontrivial generalization of the real and complex Fourier transform to quaternion cases. The four QFT components separate four cases of symmetry in real signals instead of only two in the complex FT. The QFT plays a vital role in the representation of signals and transforms a quaternion 2D signal into a quaternion-valued frequency domain signal. Many efforts had been devoted to some important properties and applications of the QFT [1-7].

In the last few years, there has been a great interest to the study of the spectrum of signals, i.e. the support of the transform of these signals relatively to certain integral transforms [8-15].

Motivated by the treatment of the QFT in quaternion algebra, in this paper we will characterize the quaternion-valued signals whose QFT has compact support. The main difficulty lies in the fact that the quaternion algebra is non-commutative, so one cannot directly extend the results for the Fourier transform to those for the QFT.

This paper is organized as follows: Section 2 is devoted to reviewing some necessary results about the quaternion algebra. In Section 3, based on the definition and some properties of the QFT, we get a result to describe the spectrum for the QFT.

\section{Preliminaries}

The quaternion algebra $\mathbb{H}$ is an extension of the algebra of complex numbers to a four dimensional real algebra. It is given by

${ }^{*}$ Corresponding author.

$$
\mathbb{H}=\left\{q \mid q_{0}+i q_{1}+j q_{2}+k q_{3}, q_{0}, q_{1}, q_{2}, q_{3} \in \mathbb{R}\right\},
$$

where the elements $i, j, k$ obey Hamilton's multiplication rules

$$
\begin{gathered}
i j=-j i=k, j k=-k j=i, k i=-i k=j \\
i^{2}=j^{2}=k^{2}=i j k=-1 .
\end{gathered}
$$

The conjugate of a quaternion $q$ is obtained by changing the sign of the pure quaternion part, i.e., $q=q_{0}-q=q_{0}-i q_{1}-j q_{2}-k q_{3}$. The modulus $|q|$ of a quaternion $q$ is defined by

$|q|=\sqrt{q \bar{q}}=\sqrt{q_{0}^{2}+q_{1}^{2}+q_{2}^{2}+q_{3}^{2}}$. Using the conjugate and the modulus of a quaternion, we can define the inverse of $q \in \mathbb{H} \backslash\{0\}$ by $q^{-1}=\frac{\bar{q}}{|q|^{2}}$ which shows that $\mathbb{H}$ is a normed division algebra. Moreover, for arbitrary $a, b \in \mathbb{H}$ the following identity holds

$$
|a b|=|a||b| \text {. }
$$

We introduce the space $L^{2}\left(\mathbb{R}^{2} ; \mathbb{H}\right)$ as the left module of all quaternion-valued functions $f: \mathbb{R}^{2} \rightarrow \mathbb{H}$ with finite norm

$$
\|f\|=\left(\int_{\mathbb{R}^{2}}|f(x)|^{2} d^{2} x\right)^{\frac{1}{2}}
$$

where $d^{2} x=d x_{1} d x_{2}$ represents the usual Lebesgue measure in $\mathbb{R}^{2}$. Moreover, denote the space $L^{1}\left(\mathbb{R}^{2} ; \mathbb{H}\right)$ the left module of all quaternion-valued functions $f: \mathbb{R}^{2} \rightarrow \mathbb{H}$ satisfying $\int_{\mathbb{R}^{2}}|f(x)| d^{2} x<\infty$.

\section{Main Results}

Note that $L^{1}\left(\mathbb{R}^{2} ; \mathbb{H}\right) \cap L^{2}\left(\mathbb{R}^{2} ; \mathbb{H}\right)$ is dense in 
$L^{2}\left(\mathbb{R}^{2} ; \mathbb{H}\right)$. Hence, standard density arguments allow us to extend the definition of the QFT of $f \in L^{1}\left(\mathbb{R}^{2} ; \mathbb{H}\right)$ in a unique way to the whole of $L^{2}\left(\mathbb{R}^{2} ; \mathbb{H}\right)$. We give the following definition of the QFT as an operator from $L^{2}\left(\mathbb{R}^{2} ; \mathbb{H}\right)$ into $L^{2}\left(\mathbb{R}^{2} ; \mathbb{H}\right)$ [3].

\section{Definition 1}

The two-sided QFT of $f \in L^{2}\left(\mathbb{R}^{2} ; \mathbb{H}\right)$ is the function $F_{q} f$ defined by

$$
F_{q} f(\omega)=\frac{1}{(2 \pi)^{2}} \int_{\mathbb{R}^{2}} e^{-i x_{1} \omega_{1}} f(x) e^{-j x_{2} \omega_{2}} d^{2} x
$$

with arbitrary frequency $\omega=\left(\omega_{1}, \omega_{2}\right)$.

The QFT can be inverted by

$$
f(x)=\frac{1}{(2 \pi)^{2}} \int_{\mathbb{R}^{2}} e^{-i x_{1} \omega_{1}} F_{q} f(\omega) e^{-j x_{2} \omega_{2}} d^{2} \omega
$$

with $d^{2} \omega=d \omega_{1} d \omega_{2}$.

In what follows, we review some properties of the QFT, such as the Parseval theorem and the partial derivative. For more details, we refer to [3].

Lemma 2

For $f \in L^{2}\left(\mathbb{R}^{2} ; \mathbb{H}\right)$ we have

$$
\|f\|=1 / 2 \pi\left\|F_{q} f\right\|,
$$

where the norm $\|\cdot\|$ is defined by Equation (2.2).

\section{Lemma 3}

If $\frac{\partial^{m+n}}{\partial x_{1}^{m} \partial x_{2}^{n}} f(x) \in L^{2}\left(\mathbb{R}^{2} ; \mathbb{H}\right), m, n \in \mathbb{N}_{0}$ and $f \in L^{2}\left(\mathbb{R}^{2} ; \mathbb{H}\right)$. Then we have

$$
F_{q}\left\{\frac{\partial^{m+n}}{\partial x_{1}^{m} \partial x_{2}^{n}} f(x)\right\}(\omega)=i^{m} \omega_{1}^{m} F_{q} f(\omega) \omega_{2}^{n} j^{n},
$$

where the QFT $F_{q} f$ is defined by Equation (3.1).

Given a multi-index $\alpha=\left(\alpha_{1}, \alpha_{2}\right) \in \mathbb{Z}_{+}^{2}$, we write as usual $|\alpha|=\alpha_{1}+\alpha_{2}, \quad D^{\alpha}=\frac{\partial^{\alpha_{1}}}{\partial x_{1}^{\alpha_{1}}} \frac{\partial^{\alpha_{2}}}{\partial x_{2}^{\alpha_{2}}}$ for the partial derivative.

Moreover, we denote by $\operatorname{supp} F_{q} f$ the support of $F_{q} f$ describing the smallest close set in $\mathbb{R}^{2}$ outside which $F_{q} f$ vanishes almost everywhere. The following theorem describes the spectrum of signals for the QFT, i.e. the compactness of the support of $F_{q} f$ by means of the norm of its partial derivative on $\mathbb{R}^{2}$.

\section{Theorem 4}

Let $f \in L^{2}\left(\mathbb{R}^{2} ; \mathbb{H}\right)$. Then the QFT $F_{q} f(\omega)$ is compactly supported in $[-\sigma, \sigma]^{2}$ if and only if partial derivatives $D^{\alpha} f \in L^{2}\left(\mathbb{R}^{2} ; \mathbb{H}\right)$,

$\omega_{1}^{\alpha_{1}} \omega_{2}^{\alpha_{2}} F_{q} f \in L^{2}\left(\mathbb{R}^{2} ; \mathbb{H}\right)$

for all $\alpha \in \mathbb{Z}_{+}^{2}$ and

$$
\lim _{|\alpha| \rightarrow \infty}\left\|D^{\alpha} f\right\|^{1 /|\alpha|}=\sigma,
$$

where $\sigma=\sup \left\{\left|\omega_{k}\right|, k=1,2: F_{q} f(\omega) \neq 0, \omega \in \mathbb{R}^{2}\right\}$.
PROOF. Firstly, we prove the necessity. Suppose that $\operatorname{supp} F_{q} f(\omega)=[-\sigma, \sigma]^{2}$. The compactness of the support of $F_{q} f$ and $f \in L^{2}\left(\mathbb{R}^{2} ; \mathbb{H}\right)$ imply that $\omega_{1}^{\alpha_{1}} \omega_{2}^{\alpha_{2}} F_{q} f$ belongs to $L^{1}\left(\mathbb{R}^{2} ; \mathbb{H}\right) \cap L^{2}\left(\mathbb{R}^{2} ; \mathbb{H}\right)$, thus partial derivatives $D^{\alpha} f$ exist and belong to $L^{2}\left(\mathbb{R}^{2} ; \mathbb{H}\right)$ for all $\alpha \in \mathbb{Z}_{+}^{2}$. Moreover, by Lemma 3 we have

$$
F_{q}\left\{D^{\alpha} f\right\}(\omega)=i^{\alpha_{1}} \omega_{1}^{\alpha_{1}} F_{q} f \omega_{2}^{\alpha_{2}} j^{\alpha_{2}} .
$$

Applying Lemma 2, it follows

$$
\left\|D^{\alpha} f\right\|^{2}=\frac{1}{(2 \pi)^{2}} \int_{\mathbb{R}^{2}}\left|i^{\alpha_{1}} \omega^{\alpha_{1}} F_{q} f(\omega) \omega_{2}^{\alpha_{2}} j^{\alpha_{2}}\right|^{2} d^{2} \omega,
$$

that is,

$$
\left\|D^{\alpha} f\right\|^{2}=\frac{1}{(2 \pi)^{2}} \int_{[-\sigma, \sigma]^{2}} \omega_{1}^{2 \alpha_{1}} \omega_{2}^{2 \alpha_{2}}\left|F_{q} f(\omega)\right|^{2} d^{2} \omega
$$

based on Equation (2.1) and $\left|i^{\alpha_{1}}\right|=\left|j^{\alpha_{2}}\right|=1$. Thus, we obtain

$$
\begin{aligned}
\left\|D^{\alpha} f\right\|^{2} & =\frac{1}{(2 \pi)^{2}} \int_{[-\sigma, \sigma]^{2}} \omega_{1}^{2 \alpha_{1}} \omega_{2}^{2 \alpha_{2}}\left|F_{q} f(\omega)\right|^{2} d^{2} \omega \\
& \leq \frac{1}{(2 \pi)^{2}} \sigma^{2|\alpha|}\left\|F_{q} f\right\|^{2}=\sigma^{2|\alpha|}\|f\|^{2},
\end{aligned}
$$

which leads to $\left\|D^{\alpha} f\right\|^{1 /|\alpha|} \leq C^{1 / \alpha \mid} \sigma$ with the constant $C=\|f\|$ independent of $|\alpha|$. Then, we have

$$
\limsup \sup _{|\alpha| \rightarrow \infty}\left\|D^{\alpha} f\right\|^{1 /|\alpha|} \leq \sigma
$$

due to $\lim _{|\alpha| \rightarrow \infty} C^{1 /|\alpha|}=1$ for all $0<C<\infty$. On the other hand, using Equation (3.2) again, for $\varepsilon \in(0, \sigma / 2)$, it holds

$$
\begin{aligned}
\left\|D^{\alpha} f\right\|^{2} & \geq \frac{1}{(2 \pi)^{2}} \int_{[\sigma-2 \varepsilon, \sigma-\varepsilon]^{2}} \omega_{1}^{2 \alpha_{1}} \omega_{2}^{2 \alpha_{2}}\left|F_{q} f(\omega)\right|^{2} d^{2} \omega \\
& \geq(\sigma-2 \varepsilon)^{2|\alpha|} \frac{1}{(2 \pi)^{2}} \int_{[\sigma-2 \varepsilon, \sigma-\varepsilon]^{2}}\left|F_{q} f(\omega)\right|^{2} d^{2} \omega,
\end{aligned}
$$

which leads to

$$
\liminf _{|\alpha| \rightarrow \infty}\left\|D^{\alpha} f\right\|^{1 / \alpha \mid} \geq \sigma-2 \varepsilon .
$$

The arbitrariness of $\varepsilon$ implies

$$
\liminf _{|\alpha| \rightarrow \infty}\left\|D^{\alpha} f\right\|^{1 /|\alpha|} \geq \sigma .
$$

Therefore, we can conclude that

$$
\lim _{|\alpha| \rightarrow \infty}\left\|D^{\alpha} f\right\|^{1 /|\alpha|}=\sigma .
$$

Secondly, we prove the sufficiency. Suppose that partial derivatives

$$
D^{\alpha} f(x) \in L^{2}\left(\mathbb{R}^{2} ; \mathbb{H}\right), \quad \omega_{1}^{\alpha_{1}} \omega_{2}^{\alpha_{2}} F_{q} f \in L^{2}\left(\mathbb{R}^{2} ; \mathbb{H}\right)
$$

for all $\alpha \in \mathbb{Z}_{+}^{2}$ and 
We need to prove that

$$
\lim _{|\alpha| \rightarrow \infty}\left\|D^{\alpha} f\right\|^{1 /|\alpha|}=d<\infty .
$$

$$
\sigma=\sup \left\{\left|\omega_{k}\right|, k=1,2: F_{q} f(\omega) \neq 0\right\},
$$

Otherwise, $F_{q} f(\omega) \neq 0$ holds for almost everywhere $\omega \in \mathbb{R}^{2}$ and thus, associated with Equation (3.2) we obtain that for arbitrary $M$ it holds

$$
\begin{aligned}
& \left\|D^{\alpha} f\right\|^{2}=\frac{1}{(2 \pi)^{2}} \int_{\mathbb{R}^{2}} \omega_{1}^{2 \alpha_{1}} \omega_{2}^{2 \alpha_{2}}\left|F_{q} f(\omega)\right|^{2} d^{2} \omega \\
& \geq \frac{1}{(2 \pi)^{2}} \int_{E} \omega_{1}^{2 \alpha_{1}} \omega_{2}^{2 \omega_{2}}\left|F_{q} f(\omega)\right|^{2} d^{2} \omega \geq C M^{2|\alpha|},
\end{aligned}
$$

where $E=\left\{\omega \in \mathbb{R}^{2}:\left|\omega_{k}\right| \geq M, k=1,2\right\}$ and $C$ is some positive constant independent of $|\alpha|$, that is to say,

$$
\left\|D^{\alpha} f\right\|^{1 /|\alpha|} \geq M \sqrt{C}^{1 /|\alpha|}
$$

The above inequality (3.4) implies

$$
\lim _{|\alpha| \rightarrow \infty}\left\|D^{\alpha} f\right\|^{1 /|\alpha|}=\infty,
$$

which contradicts the assumption (3.3). Thus, we have

$$
\sigma=\sup \left\{\left|\omega_{k}\right|, k=1,2: F_{q} f(\omega) \neq 0\right\}<\infty,
$$

which means $F_{q} f(\omega)$ is compactly supported in $[-\sigma, \sigma]^{2}$. Finally, the same technique as the part of the proof for the necessity yields that $d=\sigma$. Thus, the proof is complete.

\section{REFERENCES}

[1] S. C. Pei, J. J. Ding and J. H. Chang, "Efficient Implementation of Quaternion Fourier Transform, Convolution, and Correlation by 2-D Complex FFT," IEEE Transactions on Signal Processing, Vol. 49, No. 11, 2001, pp. 2783-2797. http://dx.doi.org/10.1109/78.960426

[2] T. Bülow, M. Felsberg and G. Sommer, "Non-Commutative Hypercomplex Fourier Transforms of Multidimensional Signals,” In: G. Sommer, Ed., Geometric Computing with Clifford Algebras, Springer, Heidelberg, 2001, pp. 187-207.

[3] E. Hitzer, "Quaternion Fourier Transform on Quaternion Fields and Generalizations,” Advances in Applied Clifford Algebras, Vol. 17, No. 3, 2007, pp. 497-517. http://dx.doi.org/10.1007/s00006-007-0037-8

[4] E. Hitzer, "Directional Uncertainty Principle for Quaternion Fourier Transform," Advances in Applied Clifford
Algebras, Vol. 20, No. 2, 2010, pp. 271-284. http://dx.doi.org/10.1007/s00006-009-0175-2

[5] S. J. Sangwine and T. A. Ell, "Hypercomplex Fourier Transforms of Color Images," IEEE Transactions on Image Processing, Vol. 16, No. 1, 2007, pp. 22-35. http://dx.doi.org/10.1109/TIP.2006.884955

[6] E. Bayro-Corrochano, N. Trujillo and M. Naranjo, “Quaternion Fourier Descriptors for Preprocessing and Recognition of Spoken Words Using Images of Spatiotemporal Representations," Journal of Mathematical Imaging and Vision, Vol. 28, No. 2, 2007, pp. 179-190. http://dx.doi.org/10.1007/s10851-007-0004-y

[7] M. Bahri, E. Hitzer, A. Hayashi and R. Ashino, "An Uncertainty Principle for Quaternion Fourier Transform," Computers \& Mathematics with Applications, Vol. 56, No. 9, 2008, pp. 2398-2410. http://dx.doi.org/10.1016/j.camwa.2008.05.032

[8] V. K. Tuan, "Spectrum of Signals," Journal of Fourier Analysis and Applications, Vol. 7, No. 3, 2001, pp. 319323. http://dx.doi.org/10.1007/BF02511817

[9] V. K. Tuan, "Paley-Wiener-Type theorems," Fractional Calculus and Applied Analysis, Vol. 2, 1999, pp. 135143.

[10] V. K. Tuan and A. I. Zayed, "Paley-Wiener-Type Theorems for a Class of Integral Transforms," Journal of Fourier Analysis and Applications, Vol. 266, 2002, pp. 200-226. http://dx.doi.org/10.1006/jmaa.2001.7740

[11] N. B. Andersen and M. De Jeu, "Real Paley-Wiener Theorems and Local Spectral Radius Formulas," Transactions of the American Mathematical Society, Vol. 362, 2010, pp. 3613-3640. http://dx.doi.org/10.1090/S0002-9947-10-05044-0

[12] N. B. Andersen, "Real Paley-Wiener Theorems for the Hankel Transform," Journal of Fourier Analysis and Applications, Vol. 12, No. 1, 2006, pp. 17-25. http://dx.doi.org/10.1007/s00041-005-4056-3

[13] Q. H. Chen, L. Q. Li and G. B. Ren, "Generalized Paley-Wiener Theorems,” International Journal of Wavelets, Multiresolution and Information, Vol. 10, No. 2, 2012, 1250020 (7 pages).

[14] C. Chettaoui, Y. Othmani and K. Trimèche, "On the Range of the Dunkl Transform on $\mathbb{R}$," Analysis and Applications, Vol. 2, No. 3, 2004, pp. 177-192. http://dx.doi.org/10.1142/S0219530504000370

[15] H. Mejjaoli and K. Trimèche, "Spectrum of functions for the Dunkl transform on $\mathbb{R}^{d}$," Fractional Calculus and Applied Analysis, Vol. 10, No. 1, 2007, pp. 19-38. 